\title{
Clinical observation of gefitinib as a first-line therapy in sixty-eight patients with advanced NSCLC
}

\author{
ZHIQIANG GAO, BAOHUI HAN, HUIMIN WANG, CHUNLEI SHI, LIWEN XIONG and AIQIN GU
}

Department of Pulmonary Medicine, Chest Hospital Affiliated to Shanghai JiaoTong University, Shanghai 200030, P.R. China

Received December 22, 2011; Accepted February 24, 2012

DOI: $10.3892 / \mathrm{ol} .2012 .631$

\begin{abstract}
The aim of this study was to evaluate the efficacy and safety of gefitinib as a first-line therapy for advanced non-small cell lung cancer (NSCLC). Patients with advanced NSCLC who did not tolerate or who refused chemotherapy received $250 \mathrm{mg} /$ day of gefitinib orally as first-line therapy. A total of 68 NSCLC patients were enrolled, and the overall response rate (RR) and disease controlled rate (DCR) with gefitinib treatment was 35.3 and $77.9 \%$, respectively. The median progression-free survival (PFS) was 6.6 months and the median survival was 15.5 months. The median PFS of the adenocarcinoma patients and the non-smokers was significantly longer compared to that of the non-adenocarcinoma patients and the smokers $(\mathrm{P}<0.05)$. The median survival of the patients with Eastern Cooperative Oncology Group Performance Status (ECOG PS) 0-1 was significantly longer compared to that of the patients with $\mathrm{PS} \geq 2$ $(\mathrm{P}<0.05)$. The most common side effects of gefitinib treatment were mild rash and diarrhea. Therefore, first-line therapy with gefitinib is an effective and tolerable treatment regimen for advanced NSCLC.
\end{abstract}

\section{Introduction}

Lung cancer has the highest incidence rate of all cancer types and is the leading cause of cancer-related mortality worldwide. Non-small cell lung cancer (NSCLC) accounts for approximately $85 \%$ of all lung cancer (1). Advanced-stage NSCLC is mainly treated with platinum-based combination treatment. The 5-year survival rate for this treatment is less than $15 \%$. Many advanced NSCLC patients with a poor general condition often cannot tolerate chemotherapy or are unwilling to undergo chemotherapy. Targeted therapy is a new technique for cancer treatment, and brings hope for advanced-stage NSCLC patients.

Correspondence to: Dr Aiqin Gu, Department of Pulmonary Medicine, Chest Hospital Affiliated to Shanghai JiaoTong University, Shanghai 200030, P.R. China

E-mail: guaiqin11@126.com

Key words: non-small cell lung cancer, gefitinib, targeted therapy, first-line therapy
Breakthrough treatments using epidermal growth factor receptor tyrosine kinase inhibitors (EGFR-TKI), including gefitinib, are often used as a second- or third-line lung cancer treatment. This type of drug therapy is currently being considered as a first-line treatment option. However, results from the INTACT and TRIBUTE trials (2-4), which combined EGFR-TKI with typical first-line treatment regimes, did not provide evidence of any survival benefit from EGFR-TKI treatment. Thus, studies are now underway to identify patient populations that may benefit from this targeted therapy as a first-line treatment.

Results from the IPASS study (5) marked gefitinib as a first-line treatment of NSCLC and opened a new era for cancer treatment. Compared to chemotherapeutics, gefitinib significantly elongated the progression-free survival (PFS) of patients with mutated EGFR genes, greatly improved safety of the treatment, and improved the quality of the life of the patients. Therefore, gefitinib is likely to become a new standard first-line treatment for NSCLC patients with mutated EGFR genes. Even when the EGFR status of patients is unknown, the demographic and clinical characteristics of patients may be used to determine which patients have a relatively high chance of benefiting from EGFR-TKI treatment as the first-line treatment strategy. In 2010, gefitinib was approved by the State Food and Drug Administration of China as a first-line treatment for locally advanced or metastatic NSCLC in patients with mutated EGFR-TK, as these patients are sensitive to gefitinib treatment.

The aim of this study was to determine the efficacy and safety of gefitinib as the first-line treatment for 68 patients with advanced NSCLC.

\section{Patients and methods}

Patients. Between September 2007 and August 2010, a total of 68 NSCLC patients who were admitted to the Shanghai Chest Hospital, Shanghai, China were recruited into the study. The patients had advanced-stage (IIIB-IV, not resectable) NSCLC and had not received any chemotherapy. Diagnoses were confirmed by bronchoscopy or CT-guided lung biopsy. Patient clinical data were collected. Of the 68 patients, 20 were male and 48 were female, with the patient age ranging from 40 to 88 years, with a median age of 71 years. Among the participants, 50 patients were smokers and 18 were non-smokers. Of the 68 patients, 60 were diagnosed with adenocarcinoma, 6 with squamous cell carcinoma and 2 with 
adenosquamous carcinoma. There were 6 patients with stage IIIB cancer and 62 with stage IV cancer. For 13 patients, the Eastern Cooperative Oncology Group Performance Status (ECOG PS) scores were between 0 and 1, and 55 patients had scores $\geq 2$. All 68 patients had at least one measurable tumor that was regularly evaluated using CT or MRI. Blood, urine and kidney function were regularly monitored through routine tests. The patients received a re-evaluation every 2 months. The last follow-up was performed on September 30, 2011.

This study was approved by the institutional review board of the Chest Hospital and written informed consent was obtained from every participant.

Treatment. Patients received $250 \mathrm{mg} /$ day gefitinib orally (AstraZeneca) until the disease progressed or intolerable toxicity occurred.

Efficacy criteria and observed parameters. Efficacy was evaluated using the Response Evaluation Criteria in Solid Tumors (RECIST) 1.1 for solid tumors (6). Each patient experienced either a complete response (CR), partial response (PR) or achieved stable disease (SD) or progressive disease (PD). The response rate (RR) was calculated using the formula: $\mathrm{RR}=\mathrm{CR}+\mathrm{PR}$. The disease control rate (DCR) was calculated as $\mathrm{DCR}=\mathrm{CR}+\mathrm{PR}+\mathrm{SD}$. Toxicity was evaluated according to the standard for adverse reactions (grades 1-4) issued by the National Cancer Institute of the USA (7). Efficacy evaluations were performed every 4 weeks, beginning 4 weeks following the initiation of treatment. Efficacy evaluations were also performed when new symptoms developed or existing symptoms worsened. PFS was defined as the time from the first drug administration to the time that evidence confirmed disease progression. The overall survival (OS) of a patient was defined as the time from the first day of oral gefitinib administration to the day the patient succumbed to the disease or the day of the patient's last follow-up visit. Performance status was scored using the ECOG PS system.

Statistical analysis. The data were analyzed with SPSS 13.0 software. Differences between the groups were tested using the $\chi^{2}$ test and the survival rates were tested using the log-rank test. $\mathrm{P}<0.05$ was considered to indicate a statistically significant difference.

\section{Results}

Short-term efficacy. Among the 68 patients, 1 patient experienced CR (1.5\%), 23 experienced PR (33.8\%), 29 achieved SD (42.6\%) and 15 achieved PD (22.1\%). The RR was $35.3 \%$ (24/68) and the DCR was $77.9 \%$ (53/68). No correlation was found between gender, age, ECOG score, pathology or smoking status and patient RR ( $>>0.05)$. However, DCR was significantly higher for adenocarcinoma patients, females and non-smoking patients compared to non-adenocarcinoma, males and smoking patients $(\mathrm{P}<0.05$; Table I).

Survival. The median PFS was 6.6 months (95\% CI 5.84-7.36 months), the median survival time (MST) was 15.5 months (95\% CI 13.56-17.44 months), and the one-year survival rate was $55.8 \%$. Treatment with gefitinib resulted in a longer median PFS in the adenocarcinoma patients compared to the non-adenocarcinoma patients (7.3 vs. 3.0 months, $\mathrm{P}=0.004)$ and in the non-smokers compared to the smokers (7.3 vs. 4.0 months, $\mathrm{P}=0.049$ ). With gefitinib treatment, the MST for patients with ECOG 0-1 was longer than that for patients with $E C O G \geq 2$ (24.6 vs. 14.8 months, $\mathrm{P}=0.041)$. No other recorded parameters, including gender, age, pathology and history of smoking were significantly correlated with patient MST (Table II and Fig. 1).

Toxicity and side effects. The most common side effect was a rash that occurred in 47 cases $(69.1 \%)$. Rashes primarily occurred on the face, chest, back, hands and feet. The rashes were mainly red pimples or acne-like rashes with or without itching. Few patients experienced skin ulceration. The majority of the rashes were resolved without treatment, but new rashes occasionally appeared. The second most common side effect was mild diarrhea ( 25 cases, $36.8 \%$ ), which was alleviated by symptomatic treatment. Anorexia was encountered in 14 cases $(20.6 \%)$, pruritus in 12 cases $(17.6 \%)$, dry skin in 11 cases $(16.2 \%)$ and oral ulcers in 8 cases $(11.2 \%)$. Abnormal liver function developed in 4 cases $(5.9 \%)$, but returned to normal following temporary withdrawal of gefitinib therapy and administration of liver function-protecting treatment for 1 week. A small number of patients experienced mild nausea or anorexia that improved following symptomatic treatment. No bone marrow suppression or renal damage was observed. There were 2 patients who developed a cough and chest tightness. CT scans of these patients indicated interstitial lung disease (ILD) and the symptoms improved following ILD treatment (Table III).

\section{Discussion}

Recently, molecular therapy targeting the EGFR has received a lot of attention from oncologists. One such therapy, the EGFR-TKI gefitinib (Iressa), has been approved in a number of countries for the treatment of advanced NSCLC (8).

A number of previous studies (9-11) have indicated the safety and effectiveness of gefitinib for the treatment of advanced NSCLC that did not respond to platinum-based chemotherapy. Gefitinib is gaining popularity as a first-line treatment for NSCLC. However, the INTACT and TRIBUTE studies (2-4), which combined EGFR-TKI and first-line chemotherapies, did not find any survival benefit for EGFR-TKI therapy. Thus, the focus was on the subpopulations that may respond well to first-line EGFR-targeting therapy. Recently, a number of studies have indicated that EGFR mutation status is key in determining the efficacy of EGFR-TKI (13). EGFR has a relatively high frequency of mutations in adenocarcinoma patients, non-smokers, females and Asians, who therefore comprise the potential target population of first-line EGFR-TKI treatment.

Publication of data from the Iressa Pan-Asia Study (IPASS) in 2008 (5) was a landmark for NSCLC treatment with gefitinib. In a randomized open-label phase III clinical trial, 1217 stage IIIB/IV Asian NSCLC patients received either gefitinib (609 patients) or carboplatin and paclitaxel (CP) (608 patients) as first-line treatment. Following 22 months of follow-up, gefitinib-treated patients had a significantly higher PFS than CP-treated patients $(\mathrm{HR}=0.741, \mathrm{P}<0.0001)$. In addition, the gefitinib group showed improvement in other factors 
Table I. Characteristics and response rates of 68 NSCLC patients undergoing gefitinib therapy.

\begin{tabular}{|c|c|c|c|c|c|c|c|c|}
\hline Characteristics & $\mathrm{n}$ & PR & $\mathrm{SD}$ & PD & $\mathrm{RR}(\%)$ & P-value & DCR $(\%)$ & P-value \\
\hline \multicolumn{9}{|l|}{ Gender } \\
\hline Male & 20 & 5 & 7 & 8 & 25.0 & \multirow[t]{2}{*}{0.251} & 60.0 & \multirow[t]{2}{*}{0.047} \\
\hline Female & 48 & 19 & 22 & 7 & 39.6 & & 85.4 & \\
\hline \multicolumn{9}{|l|}{ Age (years) } \\
\hline$<70$ & 33 & 11 & 17 & 5 & 33.3 & \multirow[t]{2}{*}{0.742} & 84.8 & \multirow[t]{2}{*}{0.182} \\
\hline$\geq 70$ & 35 & 13 & 12 & 10 & 37.1 & & 71.4 & \\
\hline \multicolumn{9}{|l|}{ ECOG score } \\
\hline $0-1$ & 13 & 5 & 7 & 1 & 38.5 & \multirow[t]{2}{*}{0.954} & 92.3 & \multirow[t]{2}{*}{0.309} \\
\hline$\geq 2$ & 55 & 19 & 22 & 14 & 34.5 & & 74.5 & \\
\hline \multicolumn{9}{|l|}{ Histology } \\
\hline Adenocarcinoma & 60 & 23 & 28 & 9 & 38.3 & \multirow[t]{2}{*}{0.297} & 85.0 & \multirow[t]{2}{*}{0.001} \\
\hline Non-adenocarcinoma & 8 & 1 & 1 & 6 & 12.5 & & 25.0 & \\
\hline \multicolumn{9}{|l|}{ Smoker } \\
\hline No & 50 & 19 & 25 & 6 & 38.0 & \multirow[t]{2}{*}{0.436} & 88.0 & \multirow[t]{2}{*}{0.003} \\
\hline Yes & 18 & 5 & 4 & 9 & 27.8 & & 50.0 & \\
\hline
\end{tabular}

NSCLC, non-small cell lung cancer; ECOG PS, Eastern Cooperative Oncology Group Performance Status; PR, partial response; SD, stable disease; $\mathrm{PD}$, progressive disease; RR, response rate; $\mathrm{DCR}$, disease control rate.

Table II. Characteristics and survival of 68 NSCLC patients undergoing gefitinib therapy.

\begin{tabular}{|c|c|c|c|c|c|}
\hline Characteristics & $\mathrm{n}$ & $\begin{array}{c}\text { Mean PFS } \\
\text { (months) }\end{array}$ & P-value & $\begin{array}{c}\text { MST } \\
\text { (months) }\end{array}$ & P-value \\
\hline \multicolumn{6}{|l|}{ Gender } \\
\hline Male & 20 & 5.0 & 0.102 & 15.7 & 0.722 \\
\hline Female & 48 & 7.2 & & 15.5 & \\
\hline \multicolumn{6}{|l|}{ Age (years) } \\
\hline$<70$ & 33 & 7.5 & 0.177 & 19.2 & 0.067 \\
\hline$\geq 70$ & 35 & 6.1 & & 14.8 & \\
\hline \multicolumn{6}{|l|}{ ECOG PS score } \\
\hline $0-1$ & 13 & 7.0 & 0.388 & 24.6 & 0.041 \\
\hline$\geq 2$ & 55 & 6.9 & & 14.8 & \\
\hline \multicolumn{6}{|l|}{ Histology } \\
\hline Adenocarcinoma & 60 & 7.3 & 0.004 & 15.8 & 0.119 \\
\hline Non-adenocarcinoma & 8 & 3.0 & & 12.4 & \\
\hline \multicolumn{6}{|l|}{ Smoker } \\
\hline No & 50 & 7.3 & 0.049 & 15.6 & 0.419 \\
\hline Yes & 18 & 4.0 & & 14.7 & \\
\hline
\end{tabular}

NSCLC, non-small cell lung cancer; ECOG PS, Eastern Cooperative Oncology Group Performance Status; PFS, progression-free survival; MST, median survival time.

compared to the $\mathrm{CP}$ group, including the objective response rate (ORR) (43.0 vs. $32.2 \%, \mathrm{P}=0.0001)$, treatment tolerability and quality of life. Stratified analysis of molecular markers revealed that $\mathrm{CP}$ treatment was similarly effective for patients with wild-type and mutant EGFR, whereas gefitinib produced an improved PFS compared to chemotherapy only in patients with mutant EGFR. Notably, $40 \%$ of patients with wild-type EGFR who received gefitinib treatment had a significantly higher risk of disease progression compared to those with mutated EGFR. However, the OS curve of the CP treatment 
$\mathbf{A}$

Progression-free survival (adenocarcinoma vs. non-adenocarcinoma)

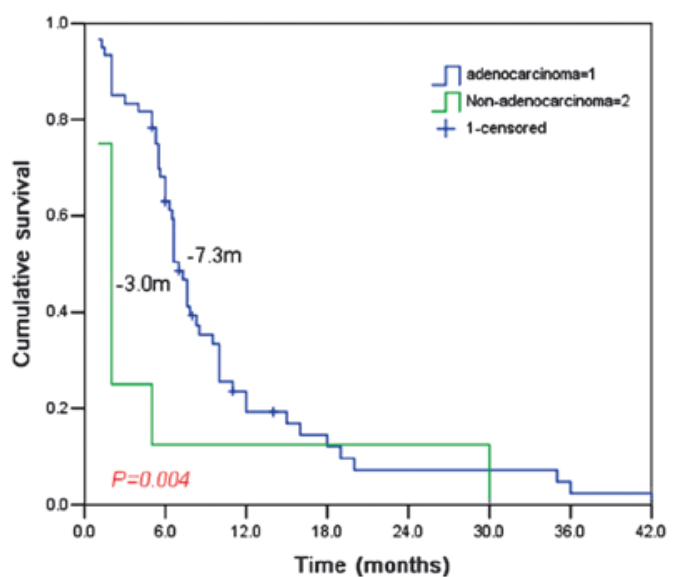

C

Survival curves (adenocarcinoma vs. non-adenocarcinoma)

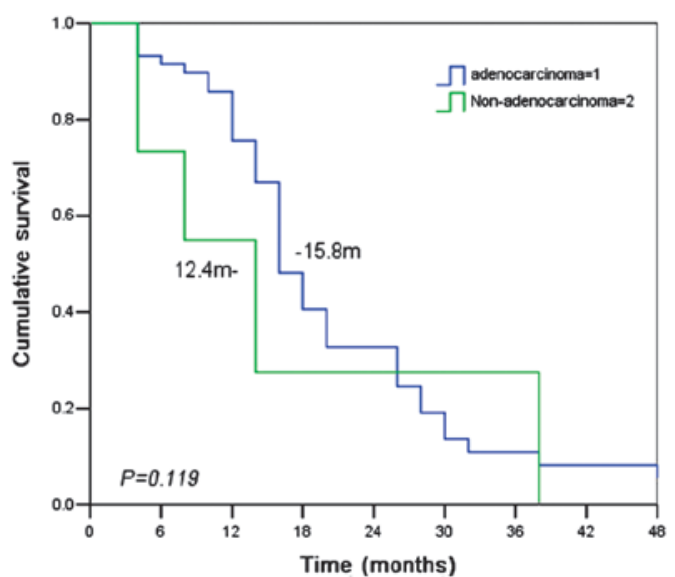

B

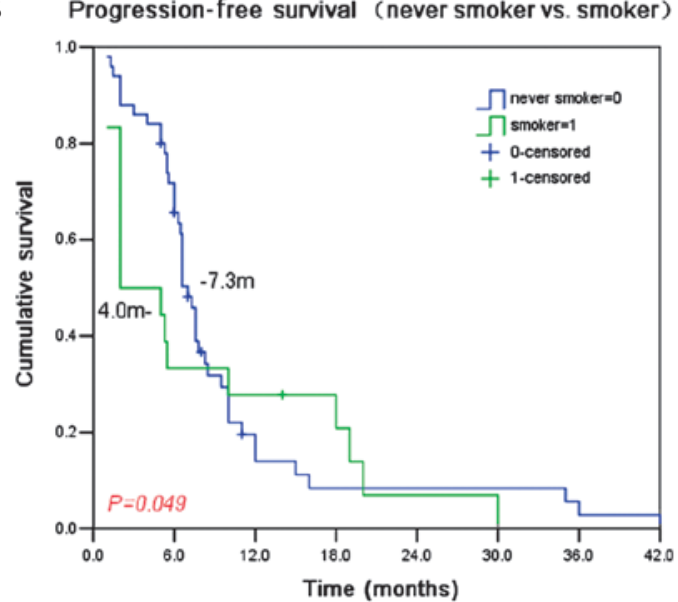

D

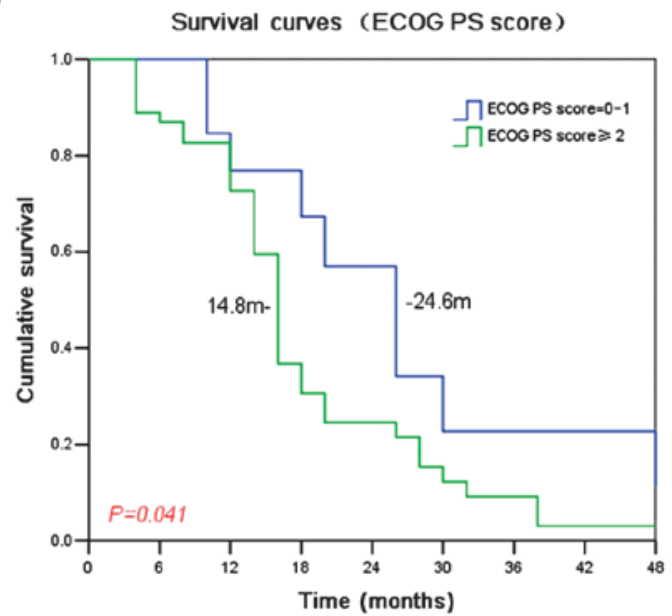

Figure 1. PFS and survival curves of NSCLC patients undergoing gefitinib treatment. Time in months is shown on the x-axis and cumulative survival on the y-axis. (A) PFS of adenocarcinoma and non-adenocarcinoma patients is shown. The curve for adenocarcinoma patients is shown in blue and the curve for nonadenocarcinoma patients is shown in green. (B) PFS of non-smokers and smokers. The curve for patients who have never smoked is shown in blue and the curve for patients who were smokers is shown in green. (C) Survival curves for adenocarcinoma and non-adenocarcinoma patients. The curve for adenocarcinoma patients is shown in blue and the curve for non-adenocarcinoma patients is shown in green. (D) Survival curves for patients with high and low ECOG PS scores. The curve for patients with scores between 0 and 1 are shown in blue, and the scores for patients with scores $\geq 2$ are shown in green. PFS, progression-free survival; NSCLC, non-small cell lung cancer.

Table III. Adverse events among 68 NSCLC patients undergoing gefitinib therapy.

\begin{tabular}{lcc}
\hline Adverse event & No. of cases (\%) & $\begin{array}{c}\text { CTC grade 3 or 4 } \\
\text { no. (\%) }\end{array}$ \\
\hline Rash & $47(69.1)$ & $2(2.9)$ \\
Diarrhea & $25(36.8)$ & 0 \\
Dry skin & $11(16.2)$ & 0 \\
Anorexia & $14(20.6)$ & $1(1.5)$ \\
Pruritus & $12(17.6)$ & 0 \\
Stomatitis & $8(11.2)$ & 0 \\
Abnormal liver & $4(5.9)$ & 0 \\
function & $3(4.4)$ & 0 \\
Nausea & $2(2.9)$ & 0 \\
Vomiting & $2(2.9)$ & 0 \\
Constipation & $1(1.5)$ & 0 \\
Alopecia & & \\
\hline
\end{tabular}

NSCLC, non-small cell lung cancer; CTC, common toxicity criteria. overlapped with that of the first-line targeting therapy, and there was no significant difference between the median OS of the 2 groups. A similarly designed study, FIRST-SIGNAL, confirmed the results of IPASS.

Although EGFR-TKI is more useful compared to other types of therapy as a first-line treatment, depending on the ethnicity, pathology and smoking history of recipients, EGFR-TKI treatment also carries an increased risk of disease progression for patients with wild-type EGFR. Results from the IPASS and the FIRST-SIGNAL studies suggested that EGFR-TKI produces a total OS comparable to that of chemotherapy when restricted to appropriate populations. Neither of these 2 first-line strategies, however, is clearly advantageous compared to the other $(12,13)$.

In this study, $88.2 \%$ of the 68 advanced NSCLCs were adenocarcinomas. In the gefitinib group, the RR was $35.3 \%$, the DCR was $77.9 \%$, the median PFS was 6.6 months, the MST was 15.5 months, and the 1 -year survival rate was $55.8 \%$. A stratified analysis indicated that the DCRs for the adenocarcinoma patients, females and non-smokers were significantly higher than those of the non-adenocarcinoma patients, males 
and smokers (all $\mathrm{P}<0.05$ ). The survival analysis demonstrated that the PFS of the adenocarcinoma patients was longer than that of the non-adenocarcinoma patients (7.3 vs. 3.0 months, $\mathrm{P}=0.004)$, and that the median PFS of the non-smokers was longer than that of the smokers (7.3 vs. 4.0 months, $\mathrm{P}=0.049$ ). The MST of the patients with ECOG 0-1 was improved compared to that of patients with ECOG $\geq 2$ (24.6 months vs. 14.8 months, $\mathrm{P}=0.041)$. Gender, age, pathology and smoking history were not significantly correlated with MST.

In this study, a number of the patients were elderly ( $\geq 70$ years of age), and some of them suffered from cardiovascular or endocrine diseases. Therefore, these patients had a relatively low tolerance to routine chemotherapy. Additionally, certain older patients were reluctant to receive chemotherapy. Gefitinib may be a good alternative as a first-line treatment for these patients, producing similar outcomes to the standard platinum-based treatment regimes. Gefitinib was, to certain extent, an effective treatment for the brain metastasis of lung cancer. In 3 of the 8 patients with brain metastasis, MRI indicated reduced tumor size in the brain 3 months following a 2-month course of gefitinib treatment. Moreover, a number of symptoms, including headache, dizziness and limb movement disorder were markedly improved.

The main adverse reactions to gefitinib treatment were rash and diarrhea; these were mostly reversible grade I-II reactions. Four patients developed abnormal liver function, which returned to normal following 1 week of liver-protecting treatment; these patients then resumed gefitinib treatment. The most serious toxic effect of gefitinib was interstitial lung disease (ILD). In this study, 2 patients suffered from cough and chest tightness, and ILD was suspected after chest CT scans were performed. These 2 patients improved following symptomatic treatment. Therefore, withdrawal of gefitinib therapy is recommended when ILD occurs.

In conclusion, gefitinib is effective for the treatment of advanced NSCLC patients, as it has minor adverse effects and is well-tolerated. Patients do not require hospitalization for this treatment. For patients who cannot tolerate or who are reluctant to receive chemotherapy, gefitinib is able to improve symptoms and quality of life, as well as provide a survival benefit, particularly for female patients, patients with adenocarcinoma and non-smokers.

\section{Acknowledgements}

This study was supported by the Shanghai Science and Technology Research Program (10140902402).

\section{References}

1. Siegel R, Ward E, Brawley O and Jemal A: Cancer statistics: the impact of eliminating socioeconomic and racial disparities on premature cancer deaths. CA Cancer J Clin 61: 212-236, 2011.

2. Giaccone G, Herbst RS, Manegold C, et al: Gefitinib in combination with gemcitabine and cisplatin in advanced non-small-cell lung cancer: a phase III trial - INTACT 1. J Clin Oncol 22: 777-784, 2004.

3. Herbst RS, Giaccone G, Schiller JH, et al: Gefitinib in combination with paclitaxel and carboplatin in advanced non-small-cell lung cancer: a phase III trial - INTACT 2. J Clin Oncol 22: 785-794, 2004.

4. Herbst RS, Prager D, Hermann R, et al: TRIBUTE: a phase III trial of erlotinib hydrochloride (OSI-774) combined with carboplatin and paclitaxel chemotherapy in advanced non-small-cell lung cancer. J Clin Oncol 23: 5892-5899, 2005.

5. Mok TS, Wu YL, Thongprasert S, et al: Gefitinib or carboplatin-paclitaxel in pulmonary adenocarcinoma. N Engl J Med 361: 947-957, 2009.

6. Eisenhauer EA, Therasse P, Bogaerts J, et al: New response evaluation criteria in solid tumours: revised RECIST guideline (version 1.1). Eur J Cancer 45: 228-247, 2009.

7. Wiffen P, Mitchel M, Snelling M and Stoner N: Oxford Handbook of Clinical Pharmacy. Oxford University Press, New York, 2007.

8. Gaughan EM and Costa DB: Genotype-driven therapies for non-small cell lung cancer: focus on EGFR, KRAS and ALK gene abnormalities. Ther Adv Med Oncol 3: 113-125, 2011.

9. Fukuoka M, Yano S, Giaccone G, et al: Multi-institutional randomized phase II trial of gefitinib for previously treated patients with advanced non-small-cell lung cancer (The IDEAL 1 Trial) [corrected]. J Clin Oncol 21: 2237-2246, 2003.

10. Kris MG, Natale RB, Herbst RS, et al: Efficacy of gefitinib, an inhibitor of the epidermal growth factor receptor tyrosine kinase, in symptomatic patients with non-small cell lung cancer: a randomized trial. JAMA 290: 2149-2158, 2003.

11. Chang A, Parikh P, Thongprasert S, et al: Gefitinib (IRESSA) in patients of Asian origin with refractory advanced non-small cell lung cancer: subset analysis from the ISEL study. J Thorac Oncol 1: 847-855, 2006.

12. Lee J, Park K, Kim S, et al: A randomized phase III study of gefitinib versus standard chemotherapy (gemcitabine plus cisplatin) as a first-line treatment for never-smokers with advanced or metastatic adenocarcinoma of the lung. J Thorac Oncol 4: S283, 2009.

13. Maemondo M, Inoue A, Kobayashi K, et al: Gefitinib or chemotherapy for non-small-cell lung cancer with mutated EGFR. N Engl J Med 362: 2380-2388, 2010. 